\title{
Cardiovascular aspects of obstructive sleep apnoea and their relevance to the assessment of the efficacy of nasal continuous positive airway pressure therapy
}

\author{
Robert J O Davies
}

Wright et $a l^{1}$ set out to assess the "health effects" of obstructive sleep apnoea (OSA) and the evidence for the efficacy of continuous positive airway pressure (CPAP) in this disorder. In their assessment they concentrated particularly on whether or not OSA causes cardiovascular disease. The review included more than 70 papers examining cardiovascular end points and less than 30 pertaining to daytime sleepiness and quality of life. It was structured to emphasise these cardiovascular issues and appeared to imply that these factors are the important reasons for the treatment of sleep apnoea. In fact this is incorrect. Patients with sleep apnoea are treated primarily to control disabling daytime sleepiness and to improve quality of life. Heated debates about possible links with cardiovascular disease continue but these are not primarily relevant to the efficacy of CPAP treatment. By getting this balance wrong, the review by Wright et al ${ }^{1}$ was seriously flawed; by asking the wrong question it inevitably produced a misleading answer.

Wright et $a l^{1}$ first dealt with "health effects" and then separately with "CPAP efficacy" and the present discussion is structured similarly. Most of the available cardiovascular evidence relates to systemic arterial blood pressure and hence this dominates what follows. The discussion of arterial blood pressure deals first with blood pressure at night in patients with OSA and then separately with daytime awake blood pressure, since the patterns and mechanisms of any blood pressure changes are different in these two states.

\section{Nocturnal hypertension}

Figure 1 shows the disturbance of blood pressure and cardiac output which occurs during obstructive apnoeas. The blood pressure trace shows repeated falls during apnoea followed by a sharp rise in the period following the apnoea which is coincident with arousal on the electroencephalogram. The magnitude of the rise in blood pressure following apnoea can be large (more than $100 \mathrm{~mm} \mathrm{Hg}$ systolic). This blood pressure rise occurs reproducibly after every apnoea and hence potentially hundreds of times throughout the night. Several clinical and basic physiological studies have reported and explained this impressive response. ${ }^{2-4}$ These show that the post-apnoeic rise is due to increasing total peripheral vascular resistance, almost certainly triggered by arousal from sleep. $^{3-5}$ The intra-apnoeic inspiratory decreases in blood pressure are due to the haemodynamic effects of obstructed inspiration. ${ }^{6}$ It is clear that these phenomena are genuine. Indeed, they are so dependably reproducible that they have been examined as possible diagnostic markers for OSA. ${ }^{7}$ Figure 2 shows the number of rises in blood pressure related to post-apnoeic arousals which occur per hour of sleep in normal sleep and OSA. There is a highly physiologically and statistically significant increase in such rises during sleep fragmented by OSA.

As well as producing blood pressure variability, these post-apnoeic increases result in a higher overall blood pressure during sleep. In a study which compared sleeping blood pressure in patients with severe OSA with that in carefully matched control subjects, OSA was associated with a rise in blood pressure of 9 $\mathrm{mm} \mathrm{Hg}^{8}$ compared with a normal fall in the control subjects. Blood pressure at night is therefore both disturbed and raised by obstructive apnoea.

Despite the solid evidence for night-time blood pressure disturbance in OSA, Wright et $a l^{1}$ ignored this area and included no reference to the published evidence demonstrating these effects, an important omission in a review of all the "health effects" of OSA which chose to concentrate on cardiovascular disease. In essential hypertension nocturnal blood pressure has been shown to be an independent adverse outcome variable associated with increased cardiovascular end organ damage. ${ }^{9}{ }^{10}$

\section{Daytime hypertension}

Those unfamiliar with the literature in this field may find the hypothesis that repetitive upper airway obstruction during sleep might lead to daytime hypertension implausible. However, animal studies have shown it is not. Brooks et $a l^{11}$ used a sophisticated canine model of obstructive apnoea to examine its effects on night-time and daytime blood pressure. In their model an ambulant dog has a tracheostomy which is closed automatically when the animal falls asleep. The airway is kept closed until the animal wakens, just as the pharyngeal airway is closed until arousal in humans. In this model OSA produces the same pattern and magnitude of night-time blood pressure disturbance as is seen in humans, ${ }^{11}$ and also generates an awake daytime mean arterial blood pressure rise to $18 \%$ above baseline. ${ }^{11}$ This daytime hypertension resolves within one month of cessation of the obstructive apnoea. ${ }^{11}$ Of course, animal data are not directly relevant to the 

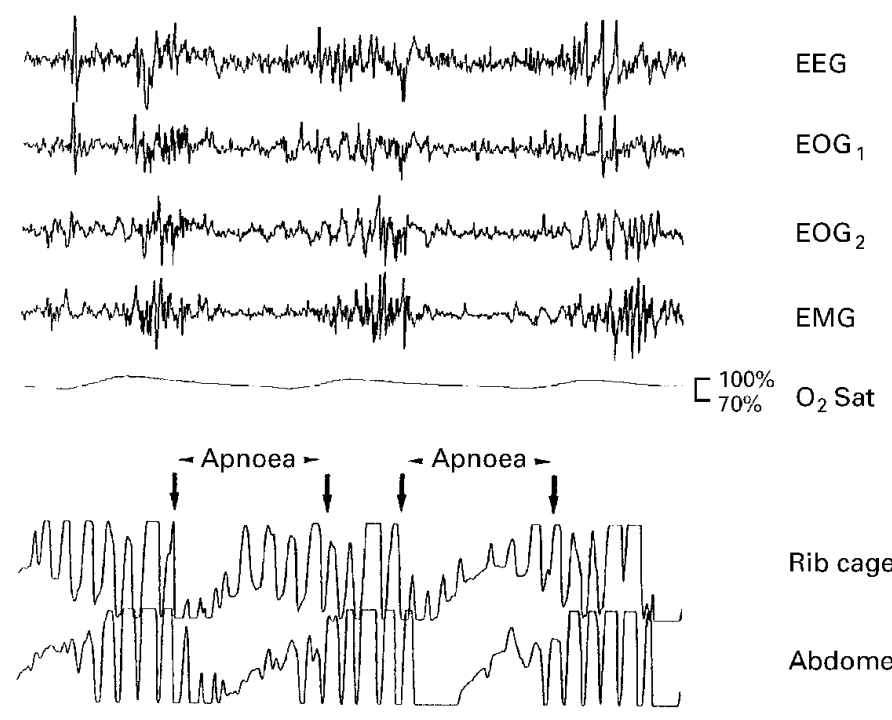

Rib cage

Abdomen

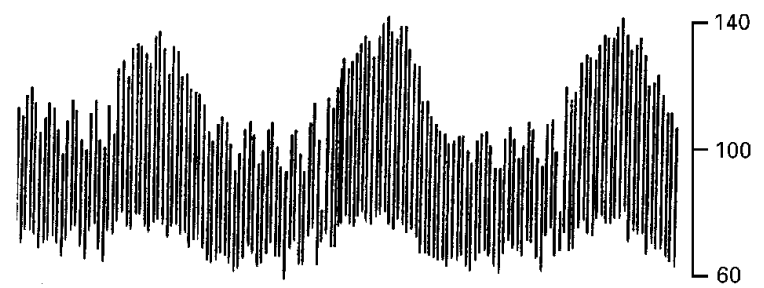

Blood pressure $(\mathrm{mm} \mathrm{Hg})$

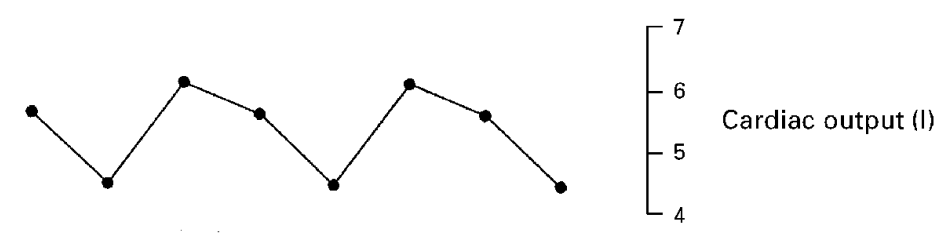

Figure 1 Typical arterial blood pressure changes during obstructive sleep apnoea. The periods of apnoea are indicated. The top trace shows the electroencephalogram with high frequency bursts indicating arousal following each apnoea. Arterial blood pressure is

represented as a single vertical line for each cardiac cycle. The line extends from the systolic to the diastolic blood pressures. The bottom trace shows cardiac output. (Data redrawn from Garpestadt et $a l^{4}$ ).

human situation, but these studies do show that a daytime hypertensive response to nighttime obstructive apnoea is consistent with mammalian physiology.

In humans the situation is less clear. All the large studies that have examined this area have found an association between raised daytime blood pressure and the presence of sleep apnoea at night. Unfortunately adult patients with sleep apnoea tend to be more overweight than normal subjects and consequently obesity and body fat distribution are important confounding variables in assessing whether this truly reflects a cause and effect relationship. In attempting to resolve this uncertainty, a series of studies using different methodologies (for example, epidemiological cross sectional surveys with statistical methods to correct confounding or case control analyses) have produced variable results. ${ }^{12}$ Some reports (including sizeable epidemiological studies) have failed to show an independent relationship while others (including one recent large

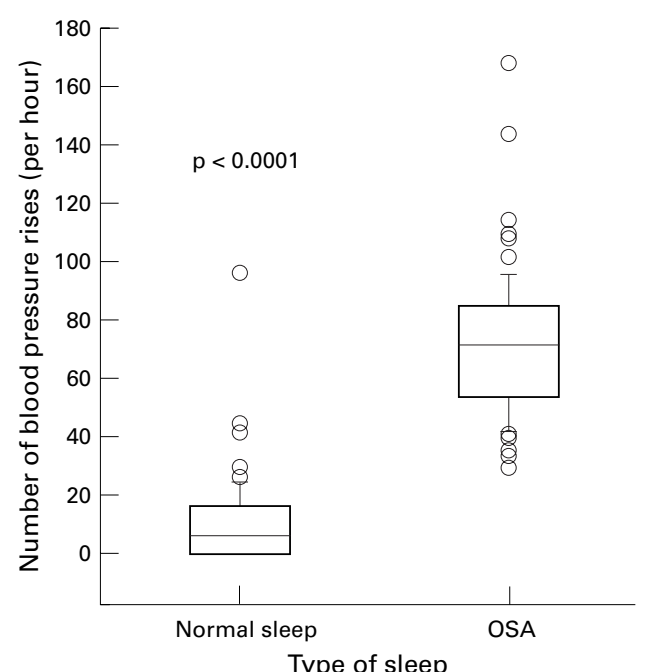

Figure 2 The number of times per hour of sleep that a blood pressure rise of the type shown in fig 1 occurred during 72 periods of normal sleep and 91 periods of obstructive sleep apnoea. The plots show the median, interquartile range 5th and 95th centiles and outlying data points. (Data drawn from Davies et $a l^{7}$ ).

and much quoted study ${ }^{14}$ ) have found a link which appears to be independent of overall obesity. The continuing uncertainty in this area means that there is, quite naturally, a broad spread of opinion in this area amongst those involved in managing sleep apnoea. ${ }^{12}{ }^{13}$ There may be a weak independent relationship between OSA and daytime blood pressure in man, and there is no doubting this relationship in the $\operatorname{dog}{ }^{11}$ but its magnitude and clinical importance remain uncertain.

\section{Effect of CPAP on blood pressure}

The evidence for the efficacy of nasal CPAP in correcting the sleeping blood pressure disturbances of OSA is unequivocal and dependably reproducible. The literature describing the use of real and sham CPAP as a research tool to switch the blood pressure changes of OSA on and off as required during studies of the physiology of these changes confirms the ability of CPAP to correct these changes reliably. ${ }^{315}$

In contrast with the undoubted efficacy of CPAP in normalising sleeping blood pressure, its efficacy in reducing awake blood pressure is not proven. Most studies that have looked at this issue have included no appropriate control groups and report variable results. ${ }^{13}$ In one small controlled crossover study of nasal CPAP compared with an oral placebo agent there was no overall effect on daytime arterial blood pressure. ${ }^{16}$ The effect of CPAP on daytime arterial blood pressure requires a further large prospective randomised and sham placebo controlled trial to assess any therapeutic effect, and without such data no case can currently be made for nasal CPAP having a useful role in reducing daytime blood pressure in patients with OSA.

\section{Other cardiovascular associations}

The literature is more unclear when other cardiovascular end organ effects such as stroke, coronary heart disease, and sudden death are 
considered. A detailed review of this literature is not appropriate for this article since here the conclusions of Wright et $a l^{1}$ are not in dispute. There are no convincing data to show that OSA is an independent risk factor for these end points after the effects of obesity, body fat distribution and tobacco intake are considered, and there are no data at all to suggest that CPAP reduces the risk of these complications.

In conclusion, an objective review of the literature pertaining to the cardiovascular effects of OSA should have found that this disorder causes repetitive blood pressure rises at the end of each obstructive apnoea, that these rises are associated with an overall rise in nocturnal blood pressure, and that these changes are reliably normalised by CPAP. There are interesting but mixed observations with regard to daytime awake arterial blood pressure, and whether there is truly an independent effect for OSA here (after allowing for obesity) remains uncertain. Even if there is an aetiological link with daytime blood pressure, there are no good data showing that this is improved by CPAP.

The relevant clinical question, therefore, is whether the nocturnal blood pressure changes which respond to nasal CPAP should themselves be seen as an indication for this therapy. In the absence of outcome data, the only way of attempting to answer this question at present is by inference from nocturnal blood pressure data in essential hypertension. Unfortunately, the characteristics and physiological mechanisms of the blood pressure disturbance of OSA are so different from those of essential hypertension that any such inference must be very suspect. The evidence does not therefore currently support the institution of CPAP for the reduction of cardiovascular risk alone, and this conclusion is consistent with current clinical practice in the UK. Nasal CPAP is prescribed primarily for the relief of disabling daytime sleepiness and the interesting but unresolved debates about the cardiovascular effects of this disorder should not be allowed to obscure this important fact.

1 Wright J, Johns R, Watt I, et al. Health effects of obstructive sleep apnoea and the effectiveness of continuous positive airways pressure: a systematic review of the research evidence. BMF 1997;314:851-60.

2 Parish JM, Shepard JW. Cardiovascular effects of sleep disorders. Chest 1990;97:1220-6.

3 Ringler J, Basner RC, Shannon R, et al. Hypoxemia alone does not explain blood pressure elevations after obstructive apneas. F Appl Physiol 1990;69:2143-8.

4 Garpestadt E, Katayama H, Parker JA, et al. Stroke volume and cardiac output decrease at termination of obstructive apneas. F Appl Physiol 1992;73:1743-8.

5 Davies RJO, Belt PJ, Robert SJ, et al. Arterial blood pressure responses to graded transient arousal from sleep in normal humans. F Appl Physiol 1993;74:1123-30.

6 Karam M, Wise RA, Natarajan TK, et al. Mechanism of decreased left ventricular stroke volume during inspiration in man. Circulation 1984;69:866-73.

7 Davies RJO, Vardi-Visy K, Clarke M, et al. Identification of sleep disruption and sleep disordered breathing from the systolic blood pressure profile. Thorax 1993;48:1242-7.

8 Davies RJO, Crosby J, Vardi-Visy K, et al. Non-invasive beat to beat arterial blood pressure during non-REM sleep in obstructive sleep apnoea and snoring. Thorax 1994;49: $335-9$.

9 Shimada K, Kawamoto A, Matsubayashi K, et al. Diurnal blood pressure variations and silent cerebrovascular damage in elderly patients with hypertension. $f$ Hypertens 1992;10:875-8.

10 O'Brien E, Sheridan J, O'Malley K. Dippers and nondippers. Lancet 1988;ii:397.

11 Brooks D, Horner RL, Kozar LF, et al. Obstructive sleep apnea as a cause of systemic hypertension. Evidence from a canine model. F Clin Invest 1997;99:106-9.

12 Silverberg DS, Oksenberg A. Essential hypertension and abnormal upper airway resistance during sleep. Sleep 1997; 20:794-805.

13 Stradling JR, Davies RJO. Sleep apnoea and hypertensionwhat a mess. Sleep 1997;20:789-93.

14 Young T, Peppard P, Palta M, et al. Population-based study of sleep-disordered breathing as a risk factor for hypertension. Arch Intern Med 1997;157:1746-52.

15 Ali NJ, Davies RJO, Fleetham JA, et al. The acute effects of continuous positive airway pressure and oxygen administration on blood pressure during obstructive sleep apnea. Chest 1992;101:1526-32.

16 Engleman HM, Gough K, Martin SE, et al. Ambulatory blood pressure on and off continuous positive airway pressure therapy for the sleep apnea/hypopnea syndrome: effects in "non-dippers". Sleep 1996;19:378-81. 